\title{
Modelo Teórico de la Célula
}

\author{
Theoretical Model of Cell \\ Juan Carlos Broncano Torres¹, Mirtha Sussan Trejo López
}

\section{RESUMEN}

Este artículo se enmarca en el ámbito de la justificación del marco teórico que la biología requiere para comprender el carácter sistémico de sus objetos de estudio, así como, de conjeturar acerca de las posibles relaciones que existe entre el todo y las partes con la finalidad de proponer nuevas líneas de investigación.

Recordemos que a inicios del siglo XX, la Biología fue fuertemente criticada por el Círculo de Viena, Por carecer de leyes, y tener un empobrecido grado de matematización en sus sistemas de clasificación y la imposibilidad de falsar muchas de sus hipótesis. (Lorenzano, 2001; 2002).

Piaget (1979) expresó en su libro Introducción a la epistemología genética que:

"Al igual que la físico-química, la biología clasifica los objetos sobre los que trabaja, dilucida sus relaciones en forma de leyes e intenta explicar causalmente estas clasificaciones y leyes. Sólo que la estructura de estas clases en lugar de alcanzar en todos los casos y con mayor o menor facilidad un nivel matemático, conserva a menudo un carácter cualitativo, o simplemente lógico".

Inmediatamente como una respuesta a este rechazo en el año 1932 se formó en Cambridge un grupo de investigadores con el nombre de "Biotheoretical Gatherings" o también "Theoretical Biology Club", y cuyos participantes principales fueron J. H. Woodger, J. Needham, C.H. Waddington, D. M. Wrinch, J. D. Bernal. Woodger, naciendo así la Biología Teórica, como un campo que defiende la necesidad de esclarecer los fundamentos teóricos de la biología.

Desde su origen se destaca por preocuparse especialmente del problema de la organización biológica y por la elaboración de herramientas metodológicas (primero matemáticas, después también computacionales) para su tratamiento científico.

Según Hempel (1965), la explicación parte de una declaración de un fenómeno a ser explicado y encuentra un conjunto de leyes ()nLLL, .....,21 y enunciados ()nEEE,....,21 en los hechos antecedentes ()nAAA,......,21 que implican una declaración.

Lorenzano (2001), destaca que la Biología carece de unicidad en el lenguaje, lo que es relativamente frecuente para numerosas ciencias:

"En lugar de encontrarnos con un único lenguaje que nos permita expresarlas [a las ciencias biológicas], nos encontramos con una gran variedad de lenguajes específicos a ciertas subdisciplinas o teorías biológicas, lo cual no elimina la posibilidad de que éstos se relacionen de algún modo entre sí".

\section{ABSTRACT}

This article is part of the scope of the justification of the theoretical framework that biology needed to understand the systemic nature of its objects of study as well as, of guessing about possible relationships between the whole and the parts in order to propose new lines of research.

Recall that in the early twentieth century, biology was strongly criticized by the Vienna Circle Lacking laws, and have a degree of mathematization impoverished in their classification systems, and the inability to falsify many of their hypotheses. (Lorenzano, 2001; 2002).

Piaget (1979) stated in his book Introduction to genetic epistemology:

"Like physical chemistry, biology classifies objects on which it works, elucidates their relations in the form of laws and attempts to explain causally these classifications and laws. Only the structure of these classes instead of achieving in all cases and more or less ease a mathematical level, often retains a qualitative, or simply logical character."

Immediately as a response to this rejection in 1932 was formed in Cambridge a group of researchers with the name "Biotheoretical Gatherings" or also "Theoretical Biology Club" and whose main participants were J. H. Woodger, J. Needham, C.H. Waddington, D. M. Wrinch, J. D. Bernal. Woodger, and thus the Theoretical Biology, as a field that defends the need to clarify the theoretical foundations of biology.

Since its origin is known for worry especially the problem of biological organization and the development of methodological tools (first mathematics, later also computer) for scientific treatment.

According to Hempel (1965), the explanation of a statement of a phenomenon to be explained and finds a set of laws ()$n L L L, \ldots \ldots, 21$ and statements ()$n E E E, \ldots \ldots, 21$ in the background facts()nAAA,......,21 that involve a statement.

Lorenzano (2001) points out that biology lacks uniqueness in language, which is relatively common to many sciences:

"Instead of meeting a unique language that allows us to express [the biological sciences], we find a variety of specific to certain sub-disciplines or biological theories languages, which does not eliminate the possibility that they are related in some way each".

\footnotetext{
${ }^{1}$ Facultad de Ciencias. Universidad Nacional José Faustino Sánchez Carrión. Lima- Perú.
} 


\section{INTRODUCCIÓN}

Los diversos planteamientos sobre la teoría del desarrollo científico se asientan en el supuesto de la invariancia del significado de los términos científicos. Por ejemplo para C. Popper el carácter teórico de todos los enunciados científicos ha llevado a abandonar la distinción tradicional absoluta entre términos teóricos y términos observacionales, sustituyéndola por una distinción, contextualizada en relación con una teoría dada, entre aquellos términos que funcionan como teóricos para la teoría en cuestión y aquellos que se consideran no teóricos en relación con la misma teoría (lo que no implica que no puedan ser teóricos en una teoría previa o diferente). Por lo que se refiere, al problema del contenido empírico o factual de las teorías científicas.

Por otro lado R. Carnap, propone una nueva concepción del pensamiento analítico de la ciencia donde se considera, reconstruir la estructura lógica de las teorías científicas de acuerdo con el ideal de la unidad de la ciencia. Sus componentes básicos son el método de reconstrucción axiomática de las teorías científicas en el marco del lenguaje formal de la lógica (predominantemente de la lógica clásica), la distinción en los lenguajes de la teoría empírica entre términos (y enunciados) teóricos y términos (y enunciados) observacionales, y finalmente el conjunto de tesis epistemológicas (fenomenalismo, empirismo, etc.) con las que se intenta resolver el problema de las relaciones entre el lenguaje de las teorías y la realidad a la que se refieren.

Por el contrario para P. Suppes se debe sustituir la axiomatización de una teoría mediante un sistema formal, al que luego se le busca una interpretación adecuada, por la definición semiformalizada de un predicado conjuntista. La idea fundamental de este nuevo enfoque de la filosofía de la ciencia se puede enunciar así: " una teoría científica consta de dos componentes principales, un núcleo $K$ y conjunto $/$ de aplicaciones propuestas de la teoría. $K$ es una estructura matemática; I es el conjunto de sistemas que constituyen modelos de".

Por tanto al analizar una teoría científica es poner de manifiesto su estructura matemática o núcleo estructural, así como localizar el conjunto de sus aplicaciones. De esta manera se combinan en la teoría de la ciencia el análisis formal y la tarea histórico-pragmática de localizar las aplicaciones paradigmáticas que constituyen un componente esencial de la teoría.

La Biología como ciencia de la vida queda enmarcada bajo esta línea de pensamiento, por tanto ella debe tener una teoría general de la vida comparable a la teoría física de los campos electromagnéticos o la teoría cuántica de las uniones químicas.

La investigación propuesta en este artículo se centra en dos aspectos fundamentales, el desarrollo de un cálculo lógico y el intento de axiomatización de cierto sector de la biología como la Citología en general y la célula en particular, así como el carácter formal de sus implicaciones esclarecedoras en el seno de la ciencia proponiendo la posibilidad de desarrollar una teorización y un tratamiento de aquellas estructuras y funciones de los seres vivos que son logizables y algeibrizables.

\section{LACÈLULA}

Según el diccionario de la RAE (Del lat. cellula, dim. de cella, hueco). f. Biol. Es la unidad fundamental de los organismos vivos, generalmente de tamaño microscópico, capaz de reproducción independiente y formada por un citoplasma y un núcleo rodeados por una membrana.

En textos especializados de Citología (Murray, 1993) y (Brown y Rothery, 1993), la célula es el vehículo a través del cual se transmite la información hereditaria que define cada especie y que además contiene la maquinaria necesaria para obtener materiales del ambiente y generar una nueva célula a su imagen, que contendrá una nueva copia de la información hereditaria.

\section{MODELO TEÓRICO DE LACÉLULA \\ A. Modelo Matemático.}

Un modelo matemático es un objeto, concepto o conjunto de relaciones, que se utiliza para representar y estudiar de forma simple y comprensible una porción de la realidad empírica.

El primer modelo matemático que se conoce data del año 1190, éste fue propuesto por Leonardo de Pisa, quien realizó demostraciones retóricas y usó segmentos de recta como representación de cantidades, a partir de entonces su alcance se ha incrementado, afinando sus usos y aplicaciones, como en la dinámica de poblaciones y disciplinas como la biología hasta llegar a la epidemiología.

\section{B. Estructura algebraica de un sistema vivo}

El libro de SNEED, The Logical Structure of Mathematical Phisics, y el de W. STEGMÜUJER, The Structure and Dynamics of Theories, son hitos importantes en la axiomatización conjuntista de las teorías empíricas, pues proporciona un método de análisis, con la intención de dar cuenta de la estructura y la dinámica de las mismas.

En el campo de las ciencias biológicas, ha sido la teoría genética la que ha utilizado este tipo de formalizaciones, como por el ejemplo el desarrollado por J.H. Woodger, en la axiomatización enunciativa de la genética mendeliana y el de W. Balzer en la axiomatización molecular.

Desarrollando así un intento de teorización y cualitativo de aquellas estructuras y funciones de los seres estableciéndose de esta manera una nueva metodología de trabajo en la biología.

La estructura interna del razonamiento biológico no está al margen como tal, y por lo tanto pensar en 
la necesidad de formación en los principios y procedimientos deductivos de la lógica no debe estar al margen de las pretensiones formativas de un biólogo.

\section{- Orgánulo}

En Biología celular es una estructura elemental, en esta axiomatización es una entidad abstracta que no consta de componentes y que es susceptible de tomar solamente dos valores que se excluyen, como son todo o nada, verdad o falsedad, sí o no, abierto o cerrado, par o impar, etc., al cual la denotaremos como: Orgcellx

\section{- Organelas celulares}

Es el conjunto de todos los orgánulos que tienen diversas especializaciones y con morfología variada. Aquí se considera como una cantidad booleana general. al cual la denotaremos como:

$$
\text { Orgcell }=\bigcup_{i \in I_{m}} \text { Orgcell }_{i}, \operatorname{con}_{m} \mathrm{C} N
$$

\section{- Citoplasma}

En biología consiste en una dispersión coloidal muy fina de aspecto granuloso cuya función es albergar los orgánulos celulares y contribuir al movimiento de estos. En esta axiomatización se considera como un sistema enlace que denotaremos como: $\mathrm{C}=$ (Orgcell, $\bar{R}$ ) en el que $\bar{R}=\left\{R_{1}, R_{2}\right\}$, siendo:

\section{$\mathrm{R}_{1}$ : Función especifica de Orgacellx.}

La función especifica de Orgcellx es el conjunto de aquellas actividades de Orgcellx que no es capaz de realizar ningún Orgacellx en $\mathrm{C}$.

\section{$\mathrm{R}_{2}$ : Proyecto teleonómico de Orgcellx.}

El proyecto teleonómico es todo aquello que contribuya al éxito de la función especifica de al éxito de la función especifica de Orgcellx.

Ademas $\bar{R}$ satisface dos axiomas:

\section{Axioma 01}

Dado Orgcellx $\bar{R}$ Orgcellz entonces Orgcellx = Orgcellz.

\section{Axioma 02}

Dado Orgcellx C, entonces existe Orgcellz $\quad$ tal que no se relacionan bajo $\bar{R}$.

\section{- Célula}

La célula se representa como la tripleta: Cell $=(C, M, R)$ en el que: $\mathrm{C}=$ (Orgcell, $\overline{\mathrm{R}})$.

M: Es el conjunto de cosas concretas, distintas de $\mathrm{C}$ que actuán sobre ella, o son afectadas por ella.
$R$ : Es el conjunto de relaciones entre los elementos de $C$ así como entre éstos y componentes de $M$, dicho conjunto incluye por lo menos una conexión.

\section{Proposición 01}

Dado el conjunto numérico $I_{n}=\{p, n<n\}$ y el conjunto de todas las células Cell, entonces la función: $\lambda: I_{n} \rightarrow$ Cell

$$
p \rightarrow \lambda(p)=\text { Cell }_{p}
$$

Es biyectiva

\section{Comunicación Celular}

Según Morgan (1989), las células de los organismos pluricelulares están provistas de mecanismos de señalamiento que les permiten recibir, procesar y responder a diferentes estímulos.

Estos mecanismos aseguran que cada célula funcione y responda a una gran diversidad de estímulos y lo haga coordinadamente con las otras células del organismo, manteniendo al organismo como entidad unitaria, a pesar de que posee millones de células distintas. Dicha coordinación sólo puede lograrse mediante una compleja red de comunicación celular.

\section{-Alfabeto Celular.}

Por la razón expuesta anteriormente definiremos el alfabeto celular como el conjunto $\Sigma=\{0,1\}$

\section{- Palabras a nivel Celular.}

Es una secuencia finita de ceros y unos seleccionados de $\Sigma$ y forman un conjunto $\Sigma^{*}$ tal que $\Sigma^{*} \quad \Sigma$.

\section{- Palabra celular vacía}

Desde que los genes están sometidos procesos de mutación y otros procesos de reorganización que provocan un cambio en la expresión fenotípica de éstos, entonces admitimos la existencia de una única cadena que no tiene símbolos, la cual denominaremos cadena vacía y será denotado por 0 .

\section{Proposición 02}

Dado el conjunto de los números naturales $\mathrm{N}$ y el conjunto de todas las palabras $\Sigma^{*}$, entonces la función:

$$
\begin{aligned}
\varphi: N & \rightarrow \Sigma^{*} \\
n & \rightarrow \varphi(n)=u_{n}
\end{aligned}
$$

\section{Es biyectiva}

\section{- Longitud de una palabra}

La longitud de una cadena $u \quad \Sigma^{*}$ se denota $\|\mathcal{u}\|$ y se define como el número de símbolos de $u$ (contando los símbolos repetidos). Es decir: 
$\|u\|=n \quad u=a_{1} * a_{2}{ }^{*} \ldots . . * a_{n} \quad a_{n} \quad \Sigma$

\section{- Concatenación de palabras}

Dado dos cadenas $u, v \quad \Sigma^{*}$, la concatenación de $u$ y $v$ se denota como $u * v$

\section{Proposición 03}

La concatenación de cadenas es una operación asociativa. Es decir $u, v, w \quad \Sigma$ * entonces $(u * v) *$ $w=u *(v * w)$.

- Lenguaje Celular L sobre el alfabeto $\Sigma$ es un subconjunto de $\Sigma^{*}$ y está definido sobre los genes en código binario contenido en los cromosomas de cada Cell

\section{- Información Celular.}

Un Modelo de Información celular (se supone la existencia de una célula emisora y receptora) es una cuádrupla $D, Q, F, R\left(q_{i}, d_{j}\right)$ donde:

- $D$ es un conjunto formado por la representación de la comunicación en la célula receptora.

- $Q$ es un conjunto formado por consultas, es decir la representación de la información que la célula emisora necesita.

- F es un marco o modelo de representación de la información, las consultas, y las relaciones existentes entre ellos.

- R $\left(q_{i}, d_{j}\right)$ es una función (ranking) que asocia un número real a cada consulta $q_{i}$ de $D$.

\section{- Posición celular}

Cell $I_{p}$ es un sistema con capacidad tanto de detección de las propiedades de su entorno, como de movimiento deberá evaluar, decidir y ejecutar los procesos necesarios con el fin de llevar a cabo su función específica.

Denotaremos por $\mathrm{x}(\mathrm{t})$ a la posición de la célula Cell $X$ en instante $t$.

\section{- Tiempo de vida celular.}

Las células de un organismo no viven indefinidamente y su vida media depende del tipo celular.

El número de células que componen un tejido en un organismo adulto permanece, dentro de ciertos límites, constante.

Algunos tipos de muerte celular conllevan la activación de mecanismos específicos que dictan que se produzca un suicidio o muerte celular programada.

Estos mecanismos fisiológicos de muerte son empleados por los organismos multicelulares durante el desarrollo, la morfogénesis y en el mantenimiento de la homeostasis tisular en el organismo adulto, así como para controlar el número de células y eliminar células infectadas, mutadas 0 dañadas. Por tanto el tiempo de existencia de $\mathrm{Ce}_{p} \mathrm{X}$ esta dado por el intervalo

$t^{p} e(x)=\left[t_{0}, t_{f}\right]$

\section{- Estado celular.}

Se entenderá por estados de una célula en un instante $t$, al conjunto de todos los genes que la célula tiene en ese instante. Denotaremos por

$x_{e}(t)=u$, donde $u=a_{1} * a_{2} * \ldots * a_{n} ; \quad a_{n} \quad \Sigma$, con

$t \quad t^{p} e(x)$. Al estado en el que se encuentra Cell en el instante $t$.

\section{Proposición 04}

Sea el conjunto $M=\left\{C e l l_{p} x /\left\|x^{p} e(t)\right\|=n\right\}$, entonces $d: M x M \quad Z^{*}$

$$
d\left(x^{p} e(t), y^{p} e(t)\right)=\Sigma\left(1-\delta_{\mathrm{x} \mathrm{e(t)y} \mathrm{e}(\mathrm{t})}(\mathrm{i})=\left\{\begin{array}{l}
1, \text { si } a_{i}=b_{j} \\
0, \text { en otros casos }
\end{array}\right.\right.
$$

у $x_{e}(t)=a_{1} * a_{2} * \ldots * a_{n} ; y_{e}(t)=b_{1} * b_{2} * \ldots . . * b_{n}$ es una métrica celular.

\section{Observación}

$d\left(x^{p} e(t) ; y^{p} e(t)\right)$ representa el número de genes de Cell $_{x p}$ no comunes a los genes de Celly respectivamente, luego cuando más pequeño es el valor de $d\left(x^{p} e(t) ; y^{p} e(t)\right)$, más grande es la coincidencia entre las cadenas genéticas de las células $\mathrm{Cell}_{p} x \quad y \mathrm{Cell}_{q} y$.

\section{CONCLUSIÓN}

El tema tratado en este artículo, es de gran valor para la enseñanza de las ciencias Biológicas. Por su efecto en la reforma de las estructuras conceptuales, así como en la concepción de la actividad investigativa, puesto que, el aspecto lingüístico, inherente a ella, se traduce en un conjunto de proposiciones sobre un ámbito concreto de objetos.

Distinguiéndose así dos tipos de objetos unos reales los cuales se nos es accesible gracias a la acción investigativa y otros ideales entendidos como constructos ideales (puramente mentales) que vienen a existir sólo como resultado de la actividad mental enmarcados en el contexto de la lógica y las matemáticas.

Así la actividad de la enseñanza-aprendizaje de las ciencias Biológicas permitirá formular algunas hipótesis fundadas en la estructura lógica matemática, cuya convalidación puede dar lugar a futuros trabajos de investigación. 


\section{REFERENCIAS BIBLIOGRÁFICAS}

Alberts, B.; Johnson, A.; Lewis, J.; Roberts, K. y Walter, P. (2010). Biología Molecular de la Célula. Omega Quinta Edición.

Attwood, T.K. y Parry-Smith, D.J. (2002). Introducción a la Bioinformática. México: Prentice Hall.

Boudon, R. (1992). Para qué sirve la noción de estructuras. Aguilar: Madrid.

Boudon, R. (1994). L'analyse mathematique des faits sociaux. París: Plon.

Brown, D. y Rothery P. (1993). Models in Biology: mathematics, statistics and computing. Chicheste: John Wiley \& Sons.

Bunge, M. (1991). General systems and holism. Dordrecht y Boston: D. Reidel Publ. Co.

Hempel, C. (1965). La explicación científica. Buenos Aires: Paidós.

Lorenzano, P. (2001). Sobre la unidad de las ciencias biológicas. Signos Filosóficos 5 (enero-junio) 121131.

Lorenzano, P. (2002). Presentación de la concepción científica del mundo: el Círculo de Viena. REDES, 9(18) 103-149.

Lwoff, B. (1994). Biological order. MIT, Cambridge,

Mass.

Lloret, M. (1996). Sistemas vivos y sus modelos matemáticos, Modelización de un ecosistema. Tesis Doctoral. Universidad de Alicante. España.

Morgan, N. G. (1989). Cell signaling. Milton Keynes, UK. Open University Press, p 1-30.

Murray, J. D. (1993). Mathematical biology. Berlín, Springer.

Piaget, J. (1979). Introducción a la epistemología genética. Buenos Aires: Paidós.

Piaget, J. (1993). El estructuralismo. Buenos Aires. Argentina: Proteo. 